\title{
Dosimetric Comparison of Sequential Versus Simultaneous-integrated Boost in Early-stage Breast Cancer Patients Treated With Breast-conserving Surgery
}

\author{
CEM ONAL, ESMA EFE, OZAN C. GULER and BERNA A. YILDIRIM \\ Department of Radiation Oncology, Baskent University Faculty of Medicine, Adana, Turkey
}

\begin{abstract}
Background/Aim: To compare simultaneousintegrated boost (SIB) versus sequential-boost $(S B)$ delivered in the context of whole-breast irradiation (WBI) via volumetricmodulated arc therapy (VMAT) and helical-tomotherapy (HT). Materials and Methods: Planning target-volume (PTV) dosimetric parameters and organs at risk $(O A R)$ were analyzed for SB plan (50 Gy plus 16 Gy boost) and SIB plan (50.4 Gy WBI and 64.4 Gy tumor bed boost) in VMAT and HT techniques. Results: Conformity and homogeneity for targetvolume doses were better in HT plans compared to VMAT plans. There were no significant differences in ipsilateral lung doses between VMAT and HT plans for SB/SIB techniques, except for a significantly higher lung V5 value with VMAT-SB, and lung V13 value with HT-SIB technique. HT provided a statistically significant decrease in contralateral lung mean V5. Conclusion: The SIB technique showed better target-volume dose distribution in both HT and VMAT plans, and better sparing heart in HT compared to the SB technique.
\end{abstract}

Breast cancer (BC) is the most common cancer and is a major cause of cancer-related death in women (1). Currently, adjuvant radiotherapy (RT), especially whole-breast irradiation (WBI) after breast-conserving surgery (BCS), is accepted as standard-of-care for early BC patients as this multimodal treatment increases local control and improves overall survival in early BC $(2,3)$. Additional tumor-bed boost after WBI has also been shown to reduce local

This article is freely accessible online.

Correspondence to: Cem Onal, MD, Prof., Baskent University Faculty of Medicine, Adana Dr. Turgut Noyan Research and Treatment Center, Department of Radiation Oncology, 01120 Adana, Turkey. Tel: +90 3223444444/1304, Fax: +90 3223444445, e-mail: hcemonal@hotmail.com

Key Words: Breast cancer, radiotherapy, helical tomotherapy, volumetric-modulated arc therapy, simultaneous integrated boost. recurrence (4); however, a long-term follow-up study found that inhomogeneous radiation in tumor-bed boosts, and use of photon beam boosts led to poorer cosmetic outcomes in patients (5). Therefore, in the past few decades, a lot of attention has focused on the development of new techniques to achieve a homogeneous dose distribution, better local control, and improve cosmetic outcomes for WBI after BCS.

Breast RT techniques range from conventional tangential field RT, field-in-field conformal RT (CRT), intensity modulated RT (IMRT), volumetric arc therapy (VMAT) to helical tomotherapy (6-11). For tumor-bed boost, relatively simple electron or hybrid techniques (i.e., a combination of different techniques for WBI and boosts), have been used. Recent dosimetric studies have demonstrated that multi-field 3D-CRT, IMRT, or VMAT are superior to electron therapy with respect to the target-volume coverage and sparing normal tissues (12-15). Moreover, simultaneous-integrated boost (SIB), which is a technique wherein a boost is delivered to the tumor bed concurrently with WBI, has been reported to be technically and dosimetrically feasible (11-13, 15-17). The rationale underlying the SIB technique is to escalate radiation doses in the area at highest risk without prolonging treatment duration. This dose escalation provides improved patient comfort while shortening the duration of RT, and also exploits the higher sensitivity of breast tumor cells to larger single doses. However, more advanced techniques are required to increase the uniformity of the radiation dose to improve patients' cosmetic outcomes and limit the doses to organs at risk (OAR) to reduce late radiation toxicities. Recently, it has been demonstrated that SIB, in combination with inversely planned IMRT, improves tumor-bed dose homogeneity and decreases normal tissue doses, when compared to 3D-CRT with sequential boost (12, 15, 18-20). Although the dosimetric advantages of SIB technique have been previously evaluated $(11,12)$, there is no strict standard for the irradiation technique used for the SIB.

Very little data are currently available on the dosimetric analysis of VMAT and HT in the context of WBI and SIB $(11,12)$. Furthermore, no study to date has compared the SIB 
technique in the context of VMAT and HT technologies. Therefore, we performed dosimetric comparisons of VMAT and HT for WBI with either an SB or a SIB in early-stage $\mathrm{BC}$ patients who had undergone BCS.

\section{Patients and Methods}

Patients. Thirty patients who received RT following BCS for early BC were enrolled for this study. Patients had stage I or II (TINOM0, T2NOM0) disease according to the 2009 7th edition of the American Joint Committee on Cancer (AJCC)/Union for International Cancer Control (UICC) staging system. In this study 15 left-sided and 15 right-sided tumors were evaluated. We included a diverse patient population having big or small breast volume, large or small tumor size, and deeply or superficially located tumors.

Delineation of target-volumes and organs at risk. All patients had undergone a $2.5-\mathrm{mm}$ slice thickness, free-breathing computed tomography (CT) scan in the supine position on $10^{\circ}-15^{\circ}$ angle breast-tilting board with both the arms elevated for treatment planning purposes.

The delineation of the target-volume and normal tissue was finally approved by two experienced radiation oncologists. Clinical targetvolume (CTV) for whole breast was delineated according to European Society for Radiotherapy and Oncology guidelines (21). The CTV included all breast parenchyma seen on planning CT. The planning target-volume for the entire breast $\left(\mathrm{PTV}_{\text {breast }}\right)$ was created by $5-\mathrm{mm}$ expansion of CTV in all directions around the tumor bed except for the skin. The anterior border of $\mathrm{PTV}_{\text {breast }}$ was trimmed from the skin by $2 \mathrm{~mm}$. The lumpectomy cavity or tumor bed was delineated according to the metal clips placed during BCS or the post-operative residual seroma. Additionally, pre-operative mammography and/or MRI images were used for better defining the $\mathrm{PTV}_{\text {boost }}$ which included the primary tumor site with a 7-mm margin in all directions within the breast tissue. The pectoralis muscles were excluded from the $\mathrm{CTV}_{\text {boost }}$ unless clinically warranted by tumor pathology. The $\mathrm{PTV}_{\text {boost }}$ was created with $5 \mathrm{~mm}$ expansion of $\mathrm{PTV}_{\text {boost }}$ in all directions. The $\mathrm{PTV}_{\text {breast }}$ was generated by subtracting $\mathrm{PTV}_{\text {boost }}$ from whole-breast volume.

In addition to the ipsilateral lung, the contralateral breast and lung, and heart were delineated on the CT image of each slice. Heart was delineated from pulmonary trunk to its most distant extent near the diaphragm, excluding pericardial fat tissue.

Treatment plans. For each patient four different plans, -VMAT-SB, VMAT-SIB, HT-SB, and HT-SIB- were generated using same CT images and the volumes delineated. For the SB technique, dose prescribed for the whole breast was 50 Gy delivered in 25 fractions, followed by a sequential boost to a total dose of 16 Gy and 2 Gy per fraction in eight fractions. For SIB technique, WBI with a total dose of $50.4 \mathrm{~Gy}, 1.8 \mathrm{~Gy}$ per fraction, with an integrated boost of 2.3 Gy per fraction (for a total dose of 64.4 Gy in 28 fractions) was delivered to the tumor bed. Both plans aimed to cover $95 \%$ of the target-volumes $\left(\mathrm{PTV}_{\text {breast }}\right.$ and $\mathrm{PTV}_{\text {boost }}$ ) with $95 \%$ of the prescribed dose. Furthermore, efforts were made to reduce the treatment volume receiving $>107 \%$ of prescribed dose to $<1 \%$.

VMAT plans were calculated with the Monaco treatment planning system version 5.0 (CMS; Elekta, Crawley, UK) using the Monte Carlo algorithm. All treatment plans were delivered with 6
MV energy Axesse linear accelerator (Elekta AB, Stockholm, Sweden) with 5-mm multileaf collimator (MLC) thickness. SB plans comprised two different plans that were optimized separately, one for whole breast receiving $50 \mathrm{~Gy}$, and other for tumor-bed boost receiving $16 \mathrm{~Gy}$. In VMAT, double ipsilateral partial arcs with a maximum individual length of $180^{\circ}$ starting from the mid-sternum, were implemented. An additional arc was generated for the tumor bed in the SB technique. The SIB technique consisted of single optimization, with the breast receiving the prescribed 50.4 Gy dose, and the tumor bed receiving $64.4 \mathrm{~Gy}$ with two different daily doses

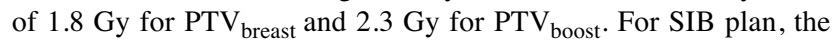
isocenter was placed on the center of $\mathrm{PTV}_{\text {boost }}$, and two arcs were generated for whole breast and boost volumes.

HT plans were generated using a Hi-Art Tomotherapy system (TomoTherapy Inc., Madison, USA), which is a helical fan-beam IMRT system equipped with inverse planning software and a 6-MV photon beam. The HT plans were made for TomoEdge Dynamic Jaws system of the TomoHDA series. A collimator aperture of 2.5 $\mathrm{cm}$, pitch of 0.287 , and modulation factor of 2.5 were used. Dose calculations were performed using the fine-dose calculation grid ( 3 $\mathrm{mm}$ in the craniocaudal direction over a $256 \times 256$ matrix in the axial plane from the original CT scan). An inverse planning optimization was used to ensure that both $\mathrm{PTV}_{\text {breast }}$ and $\mathrm{PTV}_{\text {boost }}$ receive $95 \%$ of the prescribed doses. During planning, contralateral breast, hemibody, and the posterior part of the ipsilateral side of the body were blocked.

For OARs, the dose constraints were prescribed, but an effort was made to deliver as low a dose as possible. The dose constraints prescribed for OARs were: (a) $<10 \%$ of the heart volume may receive $>30 \mathrm{~Gy}$, (b) $<20 \%$ of the ipsilateral lung may receive $>20$ $\mathrm{Gy}$, and (c) the mean dose to the contralateral breast and lung should be limited to $<5 \mathrm{~Gy}$.

Plan evaluation. For all patients, cumulative dose-volume histograms and dosimetric parameters were calculated and compared for the PTV $\mathrm{Preast}_{\text {b }}, \mathrm{PTV}_{\text {boost }}$, and OARs, for both SB and SIB plans. The D2 and D98, the minimal doses to $2 \%$ and $98 \%$ of the target-volumes, respectively, were used as surrogates for maximum and minimum doses. The volume of targetvolumes receiving 95\% (V95) and 107\% (V107) of prescribed dose was calculated. Target homogeneity and conformal index values (HI and CI, respectively) were compared. The HI was calculated as $\mathrm{HI}=[(\mathrm{D} 2-\mathrm{D} 98) / \mathrm{D} 50]$, where a greater HI value indicates poorer uniformity of the dose distribution. The CI was calculated as $\left(\mathrm{VT}_{\text {ref }} / \mathrm{VT}\right) \times\left(\mathrm{VT}_{\text {ref }} / \mathrm{Vref}\right)$, where $\mathrm{VT}_{\text {ref }}$ : targetvolume covered by isodose; VT: target-volume; $\mathrm{V}_{\text {ref: }}$ : total volume covered by $95 \%$ of isodose. The value of CI ranges from $0-1$, with a value closer to 1 indicating better conformity of the dose to the PTV.

Doses to OARs, including ipsilateral lung and heart, were analyzed. The heart doses were analyzed for entire group, and also evaluated separately for left- and right-sided tumor beds. Additionally, the doses of contralateral breast and lung were assessed. The parameters that were compared included $\mathrm{D}_{\text {mean }}$ and V5, V13, V20, and V30 for ipsilateral lung, $\mathrm{D}_{\text {mean }}, \mathrm{V} 10, \mathrm{~V} 20$, and $\mathrm{V} 30$ for heart, $\mathrm{D}_{\text {mean }}$ and V5 for contralateral breast and lung.

Statistical analysis. Statistical analyses were performed using SPSS 20.0 software (SPSS, Chicago, USA). Wilcoxon's matched-pairs test was applied to determine the significance of differences between 
volumes and doses in the VMAT and HT approaches for both SB and SIB plans. Results are presented as mean \pm standard deviation unless otherwise specified. All $p$-values reported are two-sided, and $p<0.05$ was considered statistically significant.

\section{Results}

Target-volume doses. The mean $\mathrm{PTV}_{\text {breast }}$ and $\mathrm{PTV}_{\text {boost }}$ were $986 \mathrm{~cm}^{3}\left(\right.$ range $\left.=328-1268 \mathrm{~cm}^{3}\right)$ and $68 \mathrm{~cm}^{3}($ range $=17-125$ $\mathrm{cm}^{3}$ ), respectively. Figures 1 and 2 show representative axial sections depicting the $\mathrm{PTV}_{\text {breast }}$ and $\mathrm{PTV}_{\text {breast }}$ dose distributions for VMAT and HT approaches of SB and SIB plans within a representative patient, respectively. The dosimetric parameters for target-volumes are summarized in Table I. All plans met the criteria for PTV coverage.

VMAT versus HT plans. The maximum doses (D2) for $\mathrm{PTV}_{\text {breast }}$ and PTV $\mathrm{P}_{\text {boost }}$ were significantly higher in VMATSB plan compared to HT-SB plan. The minimum dose PTV $_{\text {breast }}$ (D98) was higher in HT-SB plan compared to VMAT-SB plan; however, the minimum dose for tumor bed was higher in VMAT-SB plan compared to HT-SB plan. Although no $\mathrm{PTV}_{\text {breast }}$ and $\mathrm{PTV}_{\text {boost }}$ received more than $107 \%$ of the prescribed dose in the HT plan, mean V107 for PTV $_{\text {breast }}$ and PTV $\mathrm{V}_{\text {boost }}$ were $2.31 \%$ and $1.34 \%$, respectively, with the VMAT-SB plan. The CIs for PTV breast and PTV boost were significantly higher, and HIs were significantly lower in HT-SB compared to VMAT-SB plans.

The D2 values for PTV $\mathrm{V}_{\text {breast }}$ and PTV $\mathrm{V}_{\text {boost }}$ were significantly higher in VMAT-SIB compared to HT-SIB. Although the V95 was higher in HT-SIB plans compared to VMAT-SIB plans, it was still within planned limits. No PTV breast and PTV received more than $107 \%$ of the prescribed dose in both plans. The dose homogeneity and conformity of whole breast and tumor bed were significantly better with the HT-SIB approach than with the VMAT-SIB approach.

SIB versus SB technique. With the HT-SIB technology, the D2, D98, and V95 values for whole breast were significantly lower compared to the HT-SB technique. The dose homogeneity and conformity for tumor volumes were similar for SB/SIB plans except for the HI of PTV $\mathrm{V}_{\text {breast }}$, which was higher with $\mathrm{SB}$ technique compared to the SIB technique (0.36 vs. 0.32; $p<0.001)$.

The D2, D98, and V107 values for PTV breast and PTV $\mathrm{P}_{\text {boost }}$ with VMAT technology were significantly higher with the SB technique compared to the SIB technique. Although the $\mathrm{PTV}_{\text {breast }}$ V95 was higher in SB technique, the V95 of $\mathrm{PTV}_{\text {boost }}$ was found to be higher in SIB technique with VMAT plan. For whole breast, conformity was better with the SIB technique whereas homogeneity was better with the $\mathrm{SB}$ technique. There were no significant differences in CI and HI for tumor bed between SIB and SB techniques in VMAT plans.
OAR doses. The average dosimetric data of OARs for SB and SIB techniques using VMAT and HT plans are presented in Table II. There were no significant differences in ipsilateral lung doses between VMAT and HT approaches with SB and SIB techniques, except for significantly higher (a) lung V5 value in VMAT-SB, and (b) lung V13 value in HT-SIB. Also, there were no significant differences in ipsilateral lung and heart doses between SB and SIB techniques for both VMAT and HT technologies. The mean heart doses for all patients with right-sided tumors were significantly lower compared to left-sided tumors both in VMAT $(2.69 \pm 0.94$ Gy vs. 3.94 $\pm 1.44 \mathrm{~Gy} ; p<0.001)$ and HT plan $(2.25 \pm 0.87$ Gy vs. $4.28 \pm 1.13 \mathrm{~Gy} ; p<0.001)$ (Figure 3). Similarly, mean heart doses were significantly lower in rightsided breast and left-sided breast in SB and SIB techniques with VMAT and HT plans (Table III). For right-sided tumor, the heart doses were significantly higher in SIB technique compared to SB technique with VMAT plan $(2.80 \pm 0.88 \mathrm{~Gy}$ vs. 2.57 $\pm 1.01 \mathrm{~Gy} ; p=0.01)$. However, with HT plan, SIB plan was more advantageous in heart doses compared to SB plan $(2.13 \pm 0.69 \mathrm{~Gy} v s .2 .37 \pm 1.03 \mathrm{~Gy} ; p=0.002)$. For leftsided tumor, although no significant difference in heart doses was found between SB and SIB plans with VMAT, the mean heart doses were significantly higher in SB plan compared to those in SIB plan $(4.79 \pm 0.73$ Gy vs. $3.78 \pm 1.24 ; p<0.001)$ with HT technique.

HT provided statistically significant decreases in contralateral lung mean V5 (44\% relative decrease with SB technique and $60 \%$ relative decrease with SIB technique). Additionally, a $68 \%$ relative decrease in contralateral breast mean V5 was observed with the SB technique. A $42 \%$ relative decrease was observed with the HT-SIB technique in mean contralateral breast doses compared to the HTVMAT plan. Contralateral lung V5 was significantly lower with HT-SIB compared to the VMAT-SIB plan, and contralateral mean lung dose was lower with HT-SIB compared to VMAT-SB technique. Contralateral mean breast dose and V5 were significantly lower with the VMAT-SIB technique compared to the VMAT-SB technique.

\section{Discussion}

In the present study, we compared the target-volume coverage and dose to OARs when HT and VMAT technologies were employed in conjunction with SB and SIB techniques after BCS in early-stage BC patients. Our results showed that HT had better target-volume coverage compared to VMAT. Although there were no significant differences in dose ipsilateral lung between the two technologies, HT provided lower V5 values to contralateral breast and lung. Furthermore, SIB technique offered better target-volume dose distribution with both HT and VMAT approaches, compared to the SB technique. The mean heart doses were 


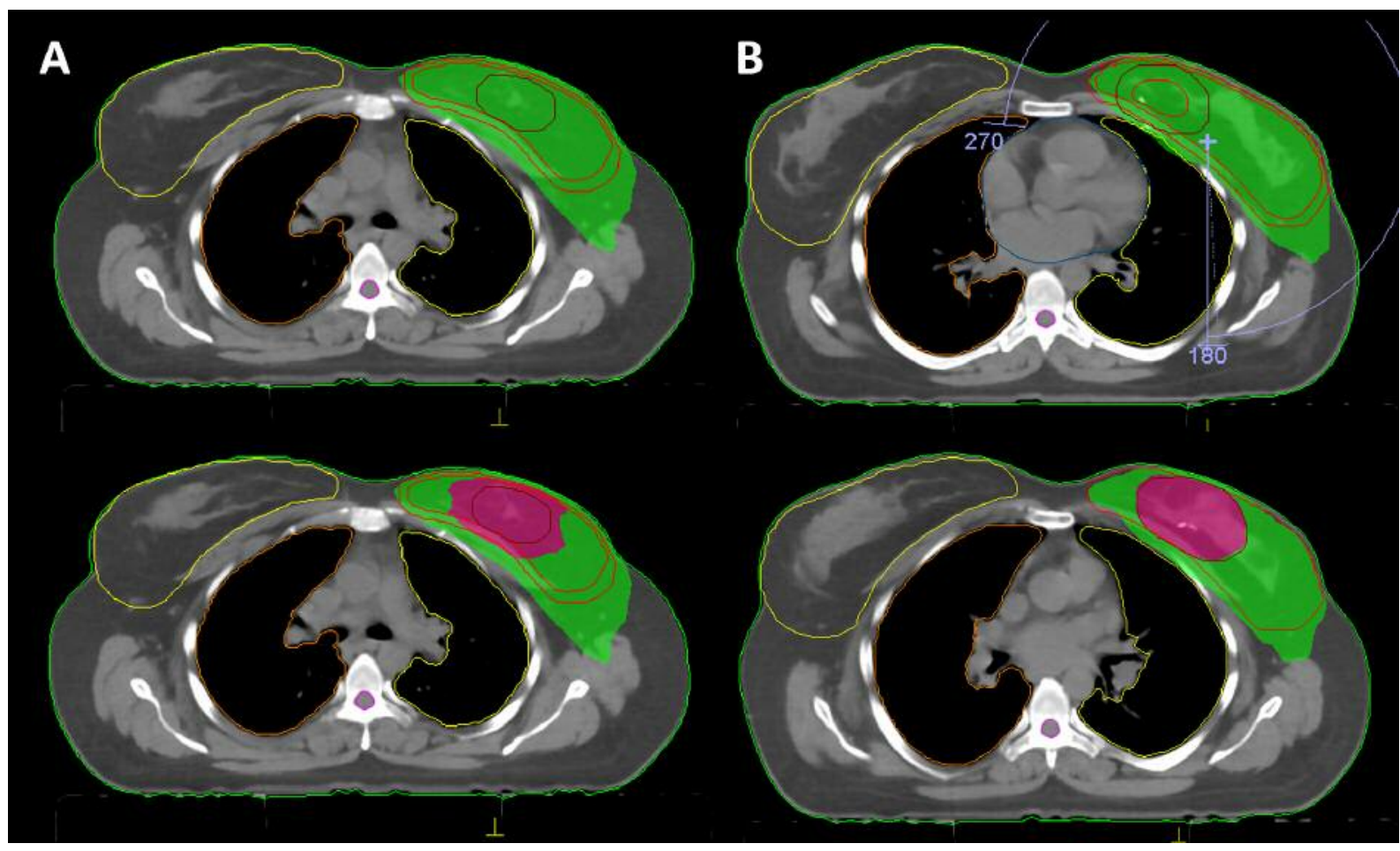

Figure 1. Dose distribution demonstrating 95\% prescribed dose of whole-breast irradiation (green area) and tumor-bed boost (magenta area) of sequential boost (A) and simultaneous-integrated boost techniques generated with VMAT plan.

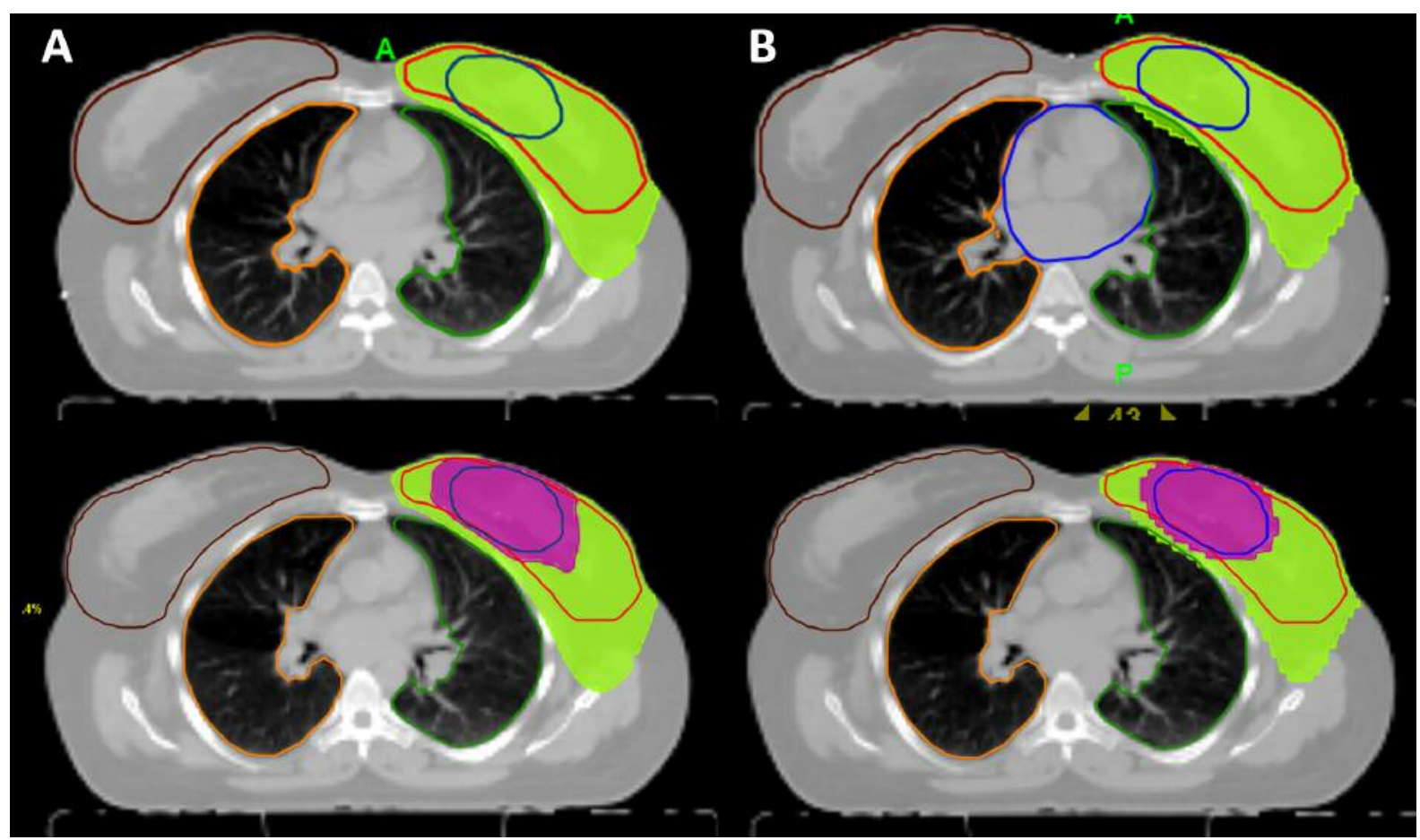

Figure 2. Dose distribution demonstrating 95\% prescribed dose of whole-breast irradiation (green area) and tumor-bed boost (magenta area) of sequential boost (A) and simultaneous-integrated boost techniques generated with HT plan. 
Table I. Target-volume doses according to treatment plans and irradiation techniques.

\begin{tabular}{|c|c|c|c|c|c|c|c|c|}
\hline \multirow[t]{3}{*}{ Parameters } & \multirow[t]{3}{*}{ HT } & \multirow{3}{*}{$\begin{array}{l}\text { VMAT } \\
\text { SB plan }\end{array}$} & \multirow[t]{3}{*}{$p$-Value } & \multirow[t]{3}{*}{ HT } & \multirow{3}{*}{$\begin{array}{l}\text { VMAT } \\
\text { SIB plan }\end{array}$} & \multirow[t]{3}{*}{$p$-Value } & \multicolumn{2}{|c|}{ SB vs. SIB plan } \\
\hline & & & & & & & HT & VMAT \\
\hline & & & & & & & $p$-Value & $p$-Value \\
\hline \multicolumn{9}{|l|}{$\mathrm{PTV}_{\text {breast }}$} \\
\hline D2 (Gy) & $53.21 \pm 0.92$ & $56.46 \pm 2.24$ & $<0.001$ & $51.80 \pm 0.71$ & $54.24 \pm 0.77$ & $<0.001$ & $<0.001$ & $<0.001$ \\
\hline D98 (Gy) & $49.96 \pm 2.34$ & $49.46 \pm 2.35$ & 0.02 & $48.66 \pm 1.70$ & $49.20 \pm 1.24$ & 0.001 & 0.003 & $<0.001$ \\
\hline V95 (\%) & $98.91 \pm 0.56$ & $99.86 \pm 0.59$ & $<0.001$ & $96.83 \pm 0.71$ & $97.80 \pm 0.64$ & 0.01 & $<0.001$ & $<0.001$ \\
\hline V107 (\%) & 0 & $2.31 \pm 1.80$ & $<0.001$ & 0 & 0 & NA & NA & $<0.001$ \\
\hline $\mathrm{CI}$ & $0.67 \pm 0.08$ & $0.63 \pm 0.07$ & $<0.001$ & $0.67 \pm 0.09$ & $0.65 \pm 0.08$ & 0.15 & 0.90 & $<0.001$ \\
\hline $\mathrm{HI}$ & $0.33 \pm 0.02$ & $0.36 \pm 0.03$ & 0.001 & $0.30 \pm 0.02$ & $0.32 \pm 0.04$ & 0.006 & $<0.001$ & 0.001 \\
\hline \multicolumn{9}{|l|}{ PTV $_{\text {boost }}$} \\
\hline D2 (Gy) & $67.96 \pm 0.68$ & $70.11 \pm 1.39$ & $<0.001$ & $66.56 \pm 0.71$ & $67.45 \pm 1.06$ & 0.002 & $<0.001$ & $<0.001$ \\
\hline D98 (Gy) & $65.42 \pm 0.62$ & $65.85 \pm 0.84$ & 0.03 & $63.82 \pm 0.37$ & $62.33 \pm 1.69$ & $<0.001$ & 0.002 & $<0.001$ \\
\hline V95 (\%) & $98.05 \pm 0.53$ & $96.52 \pm 0.70$ & $<0.001$ & $99.59 \pm 0.40$ & $98.18 \pm 1.47$ & 0.002 & $<0.001$ & $<0.001$ \\
\hline V107 (\%) & 0 & $1.34 \pm 0.88$ & $<0.001$ & 0 & 0 & NA & NA & $<0.001$ \\
\hline CI & $0.74 \pm 0.08$ & $0.72 \pm 0.08$ & 0.001 & $0.74 \pm 0.07$ & $0.71 \pm 0.07$ & $<0.001$ & 0.86 & 0.84 \\
\hline $\mathrm{HI}$ & $0.39 \pm 0.02$ & $0.41 \pm 0.03$ & $<0.001$ & $0.38 \pm 0.02$ & $0.41 \pm 0.02$ & $<0.001$ & 0.74 & 0.24 \\
\hline
\end{tabular}

Table II. Organs at risk doses according to treatment plans and irradiation technique.

\begin{tabular}{|c|c|c|c|c|c|c|c|c|}
\hline \multirow[t]{3}{*}{ Parameters } & \multirow[t]{3}{*}{ HT } & \multirow{3}{*}{$\begin{array}{l}\text { VMAT } \\
\text { SB plan }\end{array}$} & \multirow[t]{3}{*}{$p$-Value } & \multirow[t]{3}{*}{ HT } & \multirow{3}{*}{$\begin{array}{l}\text { VMAT } \\
\text { SIB plan }\end{array}$} & \multirow[t]{3}{*}{$p$-Value } & \multicolumn{2}{|c|}{ SB vs. SIB plan } \\
\hline & & & & & & & HT & VMAT \\
\hline & & & & & & & $p$-Value & $p$-Value \\
\hline \multicolumn{9}{|c|}{ Ipsilateral lung } \\
\hline V5 & $43.73 \pm 7.25$ & $53.67 \pm 7.93$ & 0.02 & $41.23 \pm 5.76$ & $43.29 \pm 3.96$ & 0.28 & 0.09 & 0.13 \\
\hline V13 & $17.39 \pm 3.68$ & $18.36 \pm 3.23$ & 0.42 & $16.57 \pm 2.28$ & $14.81 \pm 3.08$ & 0.03 & 0.32 & 0.84 \\
\hline $\mathrm{V} 20$ & $10.99 \pm 2.73$ & $11.42 \pm 2.53$ & 0.53 & $10.66 \pm 1.98$ & $9.42 \pm 2.57$ & 0.08 & 0.14 & 0.15 \\
\hline $\mathrm{V} 30$ & $6.45 \pm 2.19$ & $6.56 \pm 2.21$ & 0.83 & $5.82 \pm 1.72$ & $5.21 \pm 2.10$ & 0.26 & 0.19 & 0.07 \\
\hline Dmean & $8.66 \pm 1.43$ & $9.16 \pm 1.08$ & 0.27 & $8.11 \pm 0.73$ & $7.83 \pm 0.92$ & 0.23 & 0.05 & 0.44 \\
\hline \multicolumn{9}{|l|}{ Heart } \\
\hline $\mathrm{V} 10$ & $14.56 \pm 7.27$ & $17.22 \pm 8.46$ & 0.42 & $14.26 \pm 8.59$ & $13.63 \pm 10.23$ & 0.79 & 0.95 & 0.50 \\
\hline $\mathrm{V} 20$ & $2.30 \pm 0.42$ & $2.89 \pm 1.79$ & 0.39 & $2.47 \pm 1.44$ & $2.06 \pm 1.91$ & 0.50 & 0.09 & 0.71 \\
\hline V30 & $1.35 \pm 0.15$ & $1.56 \pm 0.71$ & 0.08 & $1.14 \pm 0.55$ & $1.11 \pm 1.06$ & 0.94 & 0.22 & 0.50 \\
\hline Mean & $3.58 \pm 1.51$ & $3.24 \pm 1.39$ & 0.21 & $2.96 \pm 1.29$ & $3.38 \pm 1.35$ & 0.02 & 0.002 & 0.64 \\
\hline \multicolumn{9}{|c|}{ Contralateral lung } \\
\hline V5 & $1.98 \pm 0.73$ & $3.52 \pm 1.40$ & 0.03 & $1.61 \pm 1.06$ & $4.04 \pm 3.22$ & 0.009 & 0.003 & 0.04 \\
\hline Dmean & $2.47 \pm 0.58$ & $2.29 \pm 0.46$ & 0.29 & $2.40 \pm 0.79$ & $2.10 \pm 0.70$ & 0.11 & 0.14 & 0.03 \\
\hline \multicolumn{9}{|c|}{ Contralateral breast } \\
\hline V5 & $1.48 \pm 1.19$ & $4.61 \pm 3.55$ & 0.03 & $1.33 \pm 0.46$ & $1.82 \pm 0.77$ & 0.64 & 0.18 & 0.02 \\
\hline Dmean & $2.35 \pm 1.15$ & $2.75 \pm 1.40$ & 0.31 & $1.83 \pm 1.57$ & $3.14 \pm 1.44$ & 0.02 & 0.88 & 0.01 \\
\hline
\end{tabular}

significantly lower for both left- and right-sided tumors using SIB technique compared to SB technique with HT plans. Additionally, the VMAT-SIB technique was advantageous in contralateral breast and lung doses over VMAT-SB technique.

With image-based RT, the PTV $\mathrm{V}_{\text {boost }}$ can be delineated on planning $\mathrm{CT}$, and the dosimetric analysis of any tumor-bed boost plan can be easily performed. The tumor-bed boost can be delivered either sequentially after WBI or with SIB technique. Previous dosimetric studies demonstrated feasibility of SIB with 3D-CRT, IMRT, and VMAT (7, 11, 12, 15-17). Furthermore, the dosimetric advantages of SIB over SB have been previously demonstrated $(14,15,22)$. Compared to the most widely used sequential electron boost, 
A

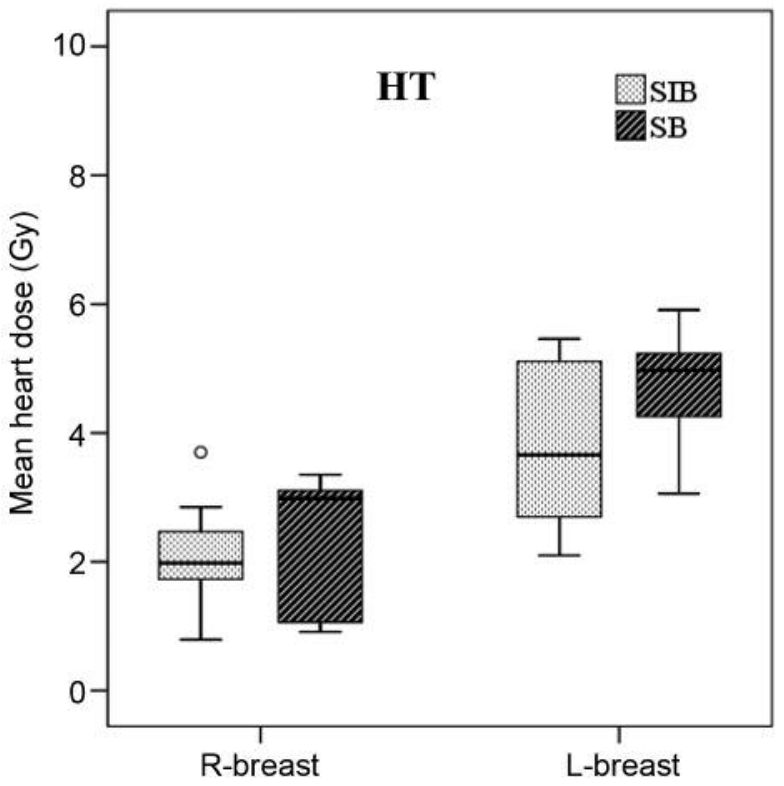

B

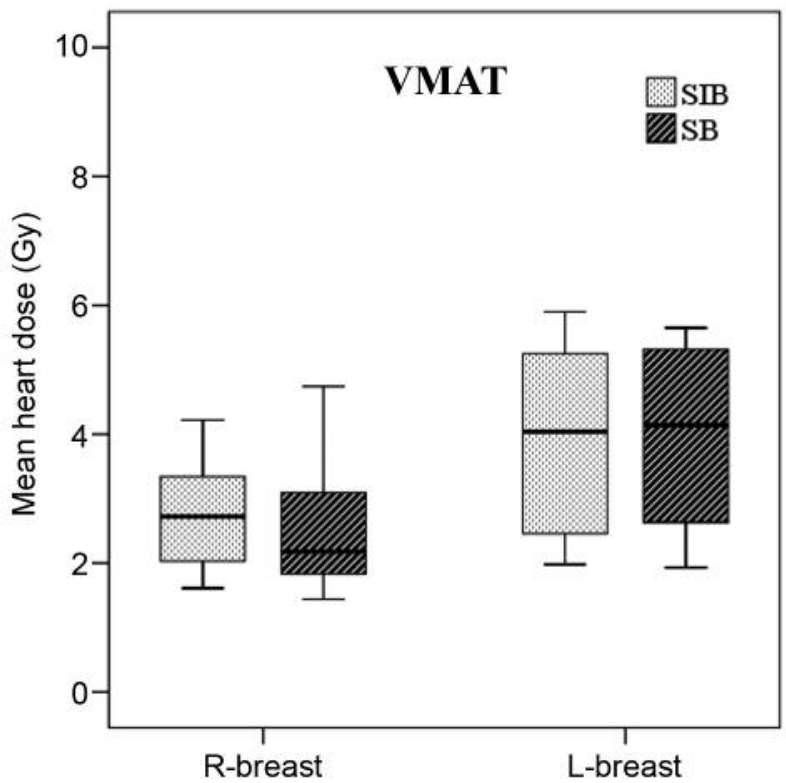

Figure 3. Box-plot graphics demonstrating the heart doses using sequential boost and simultaneous-integrated boost techniques with (A) VMAT plan and (B) HT plan for right- and left-sided breast irradiation.

SIB technique with IMRT seems to have the advantages of better skin sparing and boost volume conformity especially for deeper boost volumes $(23,24)$. In the present study, our dose distribution analysis showed more conformity and less dose spilling to the ipsilateral breast tissue outside the boost volume with the SIB technique compared to SB technique. This can be technically explained by the boost volume being surrounded by the PTV breast for the SIB technique. With the SB technique, the surrounding breast tissue has to be spared during boost irradiation in order to reduce cumulative breast doses. However, with the SIB technique, whole breast and tumor bed are simultaneously irradiated with different prescribed doses. Additionally, for the SB technique, field apertures must be several millimeters larger than the PTV $_{\text {boost }}$ volume to account for the penumbra, which causes higher doses to surrounding breast tissue. However, for the SIB technique no extra margin around the PTV $\mathrm{P}_{\text {boost }}$ is required to obtain target coverage, so the dose conformity is higher. In the current study, similar to previous studies, we found that SIB technique provides better in target-volume dose distribution compared to SB with both HT and VMAT plans. Furthermore, HT has shown superiority with absence of V107 values in SB plan, and significantly lower HI compared to VMAT in SB and SIB plans, which potentially reduces the risk of breast fibrosis.

There is no standard technique for SIB in WBI after BCS, because of conflicting results due to different RT techniques,
Table III. The heart doses according to left- and right-sided breast irradiation techniques.

\begin{tabular}{lllr}
\hline & \multicolumn{3}{c}{ Heart dose (mean $\pm \mathrm{SD})(\mathrm{Gy})$} \\
\cline { 2 - 4 } Technique & Right breast & Left breast & $p$-Value \\
\hline SB technique & & & \\
$\quad$ VMAT & $2.57 \pm 1.01$ & $3.91 \pm 1.43$ & 0.006 \\
HT & $2.37 \pm 1.03$ & $4.79 \pm 0.73$ & $<0.001$ \\
SIB technique & & & \\
$\quad$ VMAT & $2.80 \pm 0.88$ & $3.96 \pm 1.51$ & 0.02 \\
HT & $2.13 \pm 0.69$ & $3.78 \pm 1.24$ & $<0.001$ \\
\hline
\end{tabular}

treatment planning systems, and dose constraints for OARs used in these studies. Maier et al. (25) have evaluated IMRT, VMAT, and tangential arc VMAC using Monaco treatment planning for right-sided BC patients using SIB technique. The authors found that best target coverage and homogeneity was observed with VMAT, and lowest doses to the contralateral lung and breast were observed with tangential arc VMAT. Hijal et al. (11) have analyzed the dosimetric data of HT and 3DCRT with SIB technique in early-stage BC patients. The authors found that both HT and 3D-CRT provided adequate dose coverage and low heart doses; however, HT reduced higher doses within breast and decreased ipsilateral lung doses. 
Michalski et al. (12) have compared HT, IMRT, and 3D-CRT for left-sided breast tumors with SIB technique, and found that HT offered superior target-volume doses and lower doses to ipsilateral lung and heart, compared to 3D-CRT and IMRT. In dosimetric studies with more conformal techniques, VMAT $(16,25)$ and HT $(11,12)$ provide better target-volume coverage and lower lung and heart doses. In the current study comparing VMAT and HT technologies with the SIB technique, we found that HT had better target-volume coverage and provided lower doses to contralateral breast and lung compared to VMAT.

With the improved efficacy of BC therapies, the likelihood of long-term survival of patients has increased substantially. Due to the technical characteristics of IMRT, VMAT, and HT plans, radiation scattering to the surrounding normal organs is slightly higher than with conventional techniques (26). Therefore, radiation toxicities during long-term follow-up are the subject of increasing attention. Henson et al. (6) have found that the $\mathrm{BC}$ patients experienced 1.2 times the number of cardiac deaths compared to the general population. Although one study that conducted long-term follow-up in 35,000 patients undergoing RT found that the incidence of cardiovascular disease increased remarkably after RT (18), there were no increase in cardiovascular mortality in patients who underwent RT after 1980, which may be due to development/utilization of more conformal RT techniques (27). VMAT may lead to more radiation being scattered to organs due to its technical characteristics, and dosimetric studies have demonstrated that tangential field IMRT decreases ipsilateral V5 and V10 compared to multi-field IMRT and VMAT $(26,28)$. Although ipsilateral lung volumes receiving low-dose radiation were higher with the VMAT technology (suggesting that VMAT may be associated with a higher possibility of radiation-induced lung cancer), the results of long-term follow-up are still lacking. Caudrelier et al. (29) have evaluated 30 patients for long-term cardiac and pulmonary effects from mammaria interna radiotherapy using the IMRT technique. The authors concluded that V25Gy should be included in the evaluation of dose distributions besides mean heart dose with regard to long-term cardiac effects with IMRT. In the presented study, the V30Gy value was below the recommended limit of $5 \%$. Furthermore, we found that SIB technique with HT is advantageous in heart doses for both left- and right-sided tumors. Additionally, the dose levels of lung and heart in these dosimetric studies were still within limits of the 'Quantitative Analysis of Normal Tissue Effects in Clinic' (QUANTEC) guidelines $(30,31)$. According to QUANTEC guidelines, heart V25 <10\% was associated with less than $1 \%$ probability of cardiac mortality 15 years after RT (30), and the limits for lung V20 and mean lung dose were set as $<30-35 \%$ and $<20-23$ Gy, respectively (31). However further studies evaluating the long-term cardiac and pulmonary effects of VMAT and HT technologies deployed with the SIB technique are warranted.
Another important feature of VMAT and HT is the substantial contralateral breast and lung doses that raises concerns about secondary malignancies especially for earlystage BC patients. Although the V5 values of breast and lung were very low with both techniques, HT is advantageous over VMAT in sparing contralateral lung and breast, and VMAT is unsafe in terms of secondary BC, especially in young women.

This study possesses some limitations. First, our study demonstrates the dosimetric advantages of SIB technique with HT plan, however, we did not analyze radiationassociated late toxicities and long-term cosmetic outcomes of this technique. Second, we could not compare the treatment time and monitor units between VMAT and HT, since both systems are technically different. Lastly, we did not assess the inaccuracies in the setup for these complicated technologies. However, in order to improve accuracy in these complex techniques, a strike immobilization and image guidance with daily cone beam CT or breath-hold techniques are required. Notwithstanding these limitations, our dosimetric study demonstrated the applicability of SIB technique with HT plan.

\section{Conclusion}

In conclusion, our study suggests that HT may be more suitable for the SIB technique for WBI after BCS than VMAT as it offers appropriate target coverage and acceptable doses to OARs, especially the contralateral lung and breast. Furthermore, the SIB technique provided a better targetvolume dose distribution in both HT and VMAT plans, compared to the SB technique. The most important finding is that SIB technique with HT plan is better in sparing heart for both right- and left-sided tumors. Additional clinical studies with larger sample sizes are needed to assess the long-term feasibility and efficacy of SIB using HT.

\section{Conflicts of Interest}

The Authors declare no conflicts of interest regarding this study.

\section{Authors' Contributions}

Study conception and design: CO, OCG. Provision of study materials or patients: OCG, EE, BAY. Collection and assembly of data: EE, BAY. Data analysis and interpretation: CO, EE. Manuscript writing: CO, OCG. Final approval of manuscript: CO, EE, OCG, BAY. All authors read and approved the final manuscript.

\section{References}

1 Ghoncheh M, Pournamdar Z and Salehiniya H: Incidence and mortality and epidemiology of breast cancer in the world. Asian Pac J Cancer Prev 17(S3): 43-46, 2016. PMID: 27165206. DOI: 10.7314/apjcp.2016.17.s3.43 
2 Clarke M, Collins R, Darby S, Davies C, Elphinstone P, Evans V, Godwin J, Gray R, Hicks C, James S, MacKinnon E, McGale P, McHugh T, Peto R, Taylor C, Wang Y and Early Breast Cancer Trialists' Collaborative G: Effects of radiotherapy and of differences in the extent of surgery for early breast cancer on local recurrence and 15-year survival: An overview of the randomised trials. Lancet 366(9503): 2087-2106, 2005. PMID: 16360786. DOI: 10.1016/S0140-6736(05)67887-7

3 Fisher B, Anderson S, Bryant J, Margolese RG, Deutsch M, Fisher ER, Jeong JH and Wolmark N: Twenty-year follow-up of a randomized trial comparing total mastectomy, lumpectomy, and lumpectomy plus irradiation for the treatment of invasive breast cancer. N Engl J Med 347(16): 1233-1241, 2002. PMID: 12393820. DOI: 10.1056/NEJMoa022152.

4 Bartelink H, Horiot JC, Poortmans PM, Struikmans H, Van den Bogaert W, Fourquet A, Jager JJ, Hoogenraad WJ, Oei SB, Warlam-Rodenhuis CC, Pierart M and Collette L: Impact of a higher radiation dose on local control and survival in breastconserving therapy of early breast cancer: 10 -year results of the randomized boost versus no boost EORTC 22881-10882 trial. J Clin Oncol 25(22): 3259-3265, 2007. PMID: 17577015. DOI: 10.1200/JCO.2007.11.4991.

5 Immink JM, Putter H, Bartelink H, Cardoso JS, Cardoso MJ van der Hulst-Vijgen MH, Noordijk EM, Poortmans PM, Rodenhuis CC and Struikmans H: Long-term cosmetic changes after breast-conserving treatment of patients with stage I-II breast cancer and included in the EORTC 'boost versus no boost' trial. Ann Oncol 23(10): 2591-2598, 2012. PMID: 22499858. DOI: $10.1093 /$ annonc/mds066.

6 Henson KE, Reulen RC, Winter DL, Bright CJ, Fidler MM, Frobisher C, Guha J, Wong KF, Kelly J, Edgar AB, McCabe MG, Whelan J, Cutter DJ, Darby SC and Hawkins MM: Cardiac mortality among 200000 five-year survivors of cancer diagnosed at 15 to 39 years of age: The teenage and young adult cancer survivor study. Circulation 134(20): 1519-1531, 2016. PMID: 27821538. DOI: 10.1161/circulationaha.116.022514

7 Zhao H, He M, Cheng G, Han D, Wu N, Shi D, Zhao Z and Jin $\mathrm{J}$ : A comparative dosimetric study of left sided breast cancer after breast-conserving surgery treated with VMAT and IMRT. Radiat Oncol 10(231), 2015. PMID: 26577189. DOI: 10.1186/ s13014-015-0531-4.

8 Koivumaki T, Heikkila J, Vaananen A, Koskela K, Sillanmaki S and Seppala J: Flattening filter free technique in breath-hold treatments of left-sided breast cancer: The effect on beam-on time and dose distributions. Radiother Oncol 118(1): 194-198, 2016. PMID: 26709069. DOI: 10.1016/j.radonc.2015.11.032.

9 Onal C, Oymak E, Kotek A, Efe E and Arslan G: Correlation of conventional and conformal plan parameters for predicting radiation pneumonitis in patients treated with breast cancer. $\mathrm{J}$ Breast Cancer 15(3): 320-328, 2012. PMID: 26709069. DOI: $10.1016 /$ j.radonc.2015.11.032.

10 Onal C, Sonmez A, Arslan G, Oymak E, Kotek A, Efe E, Sonmez S and Dolek Y: Dosimetric comparison of the field-infield technique and tangential wedged beams for breast irradiation. Jpn J Radiol 30(3): 218-226, 2012. PMID: 221838 29. DOI: $10.1007 / \mathrm{s} 11604-011-0034-7$.

11 Hijal T, Fournier-Bidoz N, Castro-Pena P, Kirova YM, Zefkili S, Bollet MA, Dendale R, Campana F and Fourquet A: Simultaneous integrated boost in breast conserving treatment of breast cancer: A dosimetric comparison of helical tomotherapy and three-dimensional conformal radiotherapy. Radiother Oncol 94(3): 300-306, 2010. PMID: 20171752. DOI: 10.1016/j.radonc. 2009.12.043.

12 Michalski A, Atyeo J, Cox J, Rinks M, Morgia M and Lamoury G: A dosimetric comparison of 3D-CRT, IMRT, and static tomotherapy with an SIB for large and small breast volumes. Med Dosim 39(2): 163-168, 2014. PMID: 24393498. DOI: 10.1016/j.meddos.2013.12.003.

13 Son SH, Choi KH and Kim SW: Dosimetric comparison of simultaneous integrated boost with whole-breast irradiation for early breast cancer. PLoS One 12(3): e0173552, 2017. PMID: 28273157. DOI: 10.1371/journal.pone.0173552.

14 van der Laan HP, Dolsma WV, Maduro JH, Korevaar EW, Hollander M and Langendijk JA: Three-dimensional conformal simultaneously integrated boost technique for breast-conserving radiotherapy. Int J Radiat Oncol Biol Phys 68(4): 1018-1023, 2007. PMID: 17379440. DOI: 10.1016/j.ijrobp.2007.01.037.

15 Van Parijs H, Reynders T, Heuninckx K, Verellen D, Storme G and De Ridder M: Breast conserving treatment for breast cancer: Dosimetric comparison of sequential versus simultaneous integrated photon boost. Biomed Res Int 2014: 827475, 2014. PMID: 25162031. DOI: 10.1155/2014/827475.

16 Aly MM, Glatting G, Jahnke L, Wenz F and Abo-Madyan Y: Comparison of breast simultaneous integrated boost (SIB) radiotherapy techniques. Radiat Oncol 10: 139, 2015. PMID: 26156086. DOI: 10.1186/s13014-015-0452-2

17 Wu S, Lai Y, He Z, Zhou Y, Chen S, Dai M, Zhou J, Lin Q and Chi F: Dosimetric comparison of the simultaneous integrated boost in whole-breast irradiation after breast-conserving surgery: IMRT, IMRT plus an electron boost and VMAT. PLoS One 10(3): e0120811, 2015. PMID: 25781183. DOI: 10.1371/journal. pone.0120811.

18 Taylor CW, Bronnum D, Darby SC, Gagliardi G, Hall P, Jensen MB, McGale P, Nisbet A and Ewertz M: Cardiac dose estimates from Danish and Swedish breast cancer radiotherapy during 1977-2001. Radiother Oncol 100(2): 176-183, 2011. PMID: 25781183. DOI: 10.1371/journal.pone.0120811.

19 Hurkmans CW, Meijer GJ, van Vliet-Vroegindeweij C, van der Sangen MJ and Cassee J: High-dose simultaneously integrated breast boost using intensity-modulated radiotherapy and inverse optimization. Int J Radiat Oncol Biol Phys 66(3): 923-930, 2006. PMID: 16904837. DOI: 10.1016/j.ijrobp.2006.05.041.

20 Singla R, King S, Albuquerque K, Creech S and Dogan N: Simultaneous-integrated boost intensity-modulated radiation therapy (SIB-IMRT) in the treatment of early-stage left-sided breast carcinoma. Med Dosim 31(3): 190-196, 2006. PMID: 16905449. DOI: 10.1016/j.meddos.2005.11.001.

21 Offersen BV, Boersma LJ, Kirkove C, Hol S, Aznar MC, Sola AB, Kirova YM, Pignol JP, Remouchamps V, Verhoeven K, Weltens C, Arenas M, Gabrys D, Kopek N, Krause M, Lundstedt D, Marinko T, Montero A, Yarnold J and Poortmans P: ESTRO consensus guideline on target volume delineation for elective radiation therapy of early stage breast cancer, version 1.1 . Radiother Oncol 118(1): 205-208, 2016. PMID: 26791404. DOI: 10.1016/j.radonc.2015.12.027.

22 Van Parijs H, Reynders T, Heuninckx K, Verellen D, Storme G and De Ridder M: Breast conserving treatment for breast cancer: Dosimetric comparison of different non-invasive techniques for additional boost delivery. Radiat Oncol 9: 36, 2014. PMID: 24467916. DOI: 10.1186/1748-717X-9-36. 
23 Alford SL, Prassas GN, Vogelesang CR, Leggett HJ and Hamilton CS: Adjuvant breast radiotherapy using a simultaneous integrated boost: Clinical and dosimetric perspectives. J Med Imaging Radiat Oncol 57(2): 222-229, 2013. PMID: 23551785. DOI: $10.1111 /$ j.1754-9485.2012.02473.x.

24 Peters S, Schiefer H and Plasswilm L: A treatment planning study comparing Elekta VMAT and fixed field IMRT using the varian treatment planning system Eclipse. Radiat Oncol 9: 153, 2014. PMID: 25011529. DOI: 10.1186/1748-717X-9-153.

25 Maier J, Knott B, Maerz M, Loeschel R, Koelbl O and Dobler B: Simultaneous integrated boost (SIB) radiation therapy of right sided breast cancer with and without flattening filter - a treatment planning study. Radiat Oncol 11(1): 111, 2016. PMID: 27577561. DOI: 10.1186/s13014-016-0687-6

26 Otto K: Volumetric modulated arc therapy: IMRT in a single gantry arc. Med Phys 35(1): 310-317, 2008. PMID: 18293586. DOI: $10.1118 / 1.2818738$.

27 Patt DA, Goodwin JS, Kuo YF, Freeman JL, Zhang DD, Buchholz TA, Hortobagyi GN and Giordano SH: Cardiac morbidity of adjuvant radiotherapy for breast cancer. J Clin Oncol 23(30): 7475-7482, 2005. PMID: 16157933. DOI: 10.1200/JCO.2005.13.755

28 Abo-Madyan Y, Aziz MH, Aly MM, Schneider F, Sperk E, Clausen S, Giordano FA, Herskind C, Steil V, Wenz F and Glatting G: Second cancer risk after 3D-CRT, IMRT and VMAT for breast cancer. Radiother Oncol 110(3): 471-476, 2014. PMID: 24444525. DOI: 10.1016/j.radonc.2013.12.002.
29 Caudrelier JM, Meng J, Esche B, Grimard L, Ruddy T and Amjadi K: IMRT sparing of normal tissues in locoregional treatment of breast cancer. Radiat Oncol 9: 161, 2014. PMID: 25052720. DOI: 10.1186/1748-717X-9-161.

30 Gagliardi G, Constine LS, Moiseenko V, Correa C, Pierce LJ, Allen AM and Marks LB: Radiation dose-volume effects in the heart. Int J Radiat Oncol Biol Phys 76(3 Suppl): S77-85, 2010. PMID: 20171522. DOI: 10.1016/j.ijrobp.2009.04.093.

31 Marks LB, Bentzen SM, Deasy JO, Kong FM, Bradley JD, Vogelius IS, El Naqa I, Hubbs JL, Lebesque JV, Timmerman RD, Martel MK and Jackson A: Radiation dose-volume effects in the lung. Int J Radiat Oncol Biol Phys 76(3 Suppl): S70-76, 2010. PMID: 20171521. DOI: 10.1016/j.ijrobp.2009.06.091.

Received August 8, 2019

Revised August 15, 2019

Accepted August 16, 2019 